\title{
The Clinical Significance of the Expression of FENI in Primary Osteosarcoma
}

\author{
Guangxian Zhongl,* \\ Yunqing Wang ${ }^{1, *}$ \\ Hongxiang Wei ${ }^{1}$ \\ Meifang Chen ${ }^{2}$ \\ Huangfeng Lin' \\ Zhen Huang' \\ Jinlong Huang ${ }^{3}$ \\ Shenglin Wang' \\ Jianhua Lin' \\ 'Department of Orthopaedics, The First \\ Affiliated Hospital of Fujian Medical \\ University, Fuzhou, 350004, People's \\ Republic of China; ${ }^{2}$ The Health \\ Management Center, The First Affiliated \\ Hospital of Fujian Medical University, \\ Fuzhou, 350004, People's Republic of \\ China; ${ }^{3}$ Department of Hematology, The \\ First Affiliated Hospital of Fujian Medical \\ University, Fuzhou, 350004, People's \\ Republic of China
}

*These authors contributed equally to this work.

Correspondence: Jianhua Lin; Shenglin Wang

$\mathrm{Tel} / \mathrm{Fax}+8659187981029$

Email jianhual@।26.com;

Shenglinwang0216@163.com
Purpose: The aim of this research was to investigate the clinical significance of the expression of flap structure-specific endonuclease 1 (FEN1) in primary osteosarcoma.

Methods: The expression of FEN1 was detected by immunohistochemistry analysis. The association of the expression of FEN1 in osteosarcoma with clinicopathological parameters was analyzed by using $\chi^{2}$ test or Fisher's exact test. Survival analyses were performed by Kaplan-Meier method and Cox proportional hazards regression model.

Results: Of the 40 osteosarcoma patients, 19 (47.5\%) patients presented with FEN1 high expression, while in the non-neoplastic bone specimens, the FEN1 high expression was observed in $10 \%(3 / 30)$, the positive expression rate in osteosarcoma patients was significantly higher than that of non-neoplastic bone specimens $(P<0.01)$. Univariate analysis indicated that the progression-free survival (PFS) and overall survival (OS) were correlated with the expression level of FEN1 (PFS, $\mathrm{P}<0.001$; OS, $\mathrm{P}=0.002$ ), Enneking staging (PFS, $\mathrm{P}=0.026$; OS, $\mathrm{P}=0.044$ ) and chemotherapy response (PFS, $\mathrm{P}=0.019$; OS, $\mathrm{P}=0.031$ ). Multivariate analysis demonstrated that FEN1 expression was an independent prognostic factor for the PFS $(\mathrm{HR}=4.73, \mathrm{P}=0.002)$ and $\mathrm{OS}(\mathrm{HR}=4.01, \mathrm{P}=0.038)$ of osteosarcoma patients.

Conclusion: This study showed that FEN1 was overexpressed in osteosarcoma patients and positively associated with poor prognosis of osteosarcoma patients. Further studies should focus on the relative mechanisms and the targeted FEN1 therapies for osteosarcoma.

Keywords: osteosarcoma, flap endonuclease 1, immunohistochemistry, prognosis

\section{Background}

Osteosarcoma, derived from bone-forming mesenchymal cells, is the most common primary bone cancer which usually affects the children and adolescents. ${ }^{1-3}$ The standard treatment for osteosarcoma consisting of surgery and chemotherapy. ${ }^{4}$ With the introduction of neoadjuvant chemotherapy, the 5-year survival rate has improved to $60-70 \% .^{5}$ However, there is still an approximately $30 \%$ mortality in osteosarcoma patients due to the metastasis and chemotherapy resistance. ${ }^{6,7}$ Therefore, it is great significance to investigate some potential prognostic biomarkers which could be used as the targets in the development of effective agents for the osteosarcoma treatment.

Flap structure-specific endonuclease 1 (FEN1) is a member of the $\operatorname{Rad} 2$ structure-specific nuclease family, which plays an important role in Okazaki fragment maturation, stalled replication fork rescue, telomere maintenance, longpatch base excision repair, and apoptotic DNA fragmentation. ${ }^{8-10}$ Several studies have reported that overexpressed FEN1 was associated with cancer 
progression and chemotherapy resistance, and it was considered to be a marker for metastasis and poor prognosis in multiple cancers. ${ }^{11-14}$ For example, Zeng et al reported that FEN1 mediated miR-200a methylation and promoted breast cancer cell growth via MET and EGFR signaling pathways. ${ }^{15}$ Lam et al demonstrated that FEN1 was overexpressed in prostate cancer and was associated with a high Gleason score. ${ }^{16}$ Zhao et al demonstrated that miR-134-3p could inhibit the progression of ovarian cancer by modulating the overexpressed FEN1 in ovarian cancer. ${ }^{17}$ Dong et al considered miR-193b/FEN1 as a potential new target for OS treatment with the main purpose of inducing apoptosis. ${ }^{18}$ However, the relationship between the FEN1 and the clinicopathological and prognostic values of osteosarcoma was still limitedly investigated.

In the present study, the immunohistochemistry was employed to evaluate the expression levels of FEN1 in osteosarcoma, and the associations of FEN1 expression with clinicopathological parameters, progression-free survival (PFS) and overall survival (OS) time in osteosarcoma patients were further investigated, which hope to explore a new biomarker for predicting the prognosis of osteosarcoma.

\section{Materials and Methods}

\section{Patients}

A total of 40 patients who underwent neoadjuvant chemotherapy, surgical resection and postoperative chemotherapy with doxorubicin, cisplatin and ifosfamide at the Department of Orthopedics, the First Affiliated Hospital of Fujian Medical University (Fuzhou, China) between 1 January 2014, and 31 December 2017, were selected retrospectively. The general information of the collected patients was obtained from hospital records, including sex, age, tumor size and location, Enneking stage, chemotherapy response, follow-up duration and survival outcome. Forty tumor formalin-fixed paraffinembedded (FFPE) tumor samples undergoing neoadjuvant chemotherapy were obtained from the Department of Pathology for protein detection, with 30 non-neoplastic bone specimens selected between 2015 and 2016 were used as controls.

After surgical resection, all patients were monitored by using X-ray, lung CT scans and/or bone scanning every 3 months in the first 3 years and every 6 months thereafter, in order to evaluate local recurrence and distant metastasis status. PFS and OS time were defined as the interval between the date of diagnosis and the date of first tumor progression and death, respectively. Patients were censored at the date of the last follow-up if tumor progression or death had not occurred.

All participants involved in our study were informed of the aims of the study and signed informed consent forms (patients under 18 years of age were signed by their parents or legal guardian). The study protocol followed the ethical guidelines of the Declaration of Helsinki and was approved by the Ethics Committee of our institution.

\section{Immunohistochemistry (IHC) Analysis}

Osteosarcoma and non-neoplastic bone samples were examined for the expression levels of FEN1 by IHC analysis. Firstly, the paraffin-embedded tissue sections (4- $\mu \mathrm{m}$ thick) were mounted on charged glass slides for deparaffinage in xylene, rehydration in a graded alcohol series and incubation with $3 \%$ hydrogen peroxide for $10 \mathrm{~min}$ at room temperature to block endogenous peroxidase activity. Then the sections were microwaved in citrate buffer ( $\mathrm{pH}$ 6.0) for $2 \mathrm{~min}$ and then naturally cooled to room temperature for antigen retrieval. After that, the sections were incubated with rabbit polyclonal anti-FEN1 antibody (ab133311, 1:250 dilution, Abcam, USA) overnight at $4^{\circ} \mathrm{C}$. After two washes in phosphate-buffered saline (PBS), the sections were incubated with poly peroxidase-anti-mouse/rabbit IgG for $30 \mathrm{~min}$ at room temperature and then diaminobenzidine (DAB) for $5 \mathrm{~min}$ at room temperature. The immunohistochemical signals of sections were assessed by two pathologists under a light microscope (Axioskop 40; Zeiss GmbH, Jena, Germany) at five random fields independently. The FEN1 expression was scored according to the mean signal intensity $(0$, negative; 1 , weak staining; 2 , moderate staining; and 3, strong staining) and the positively stained percentage of tumor cells $(0,0-5 \% ; 1,6-25 \% ; 2,26-50 \% ; 3,51-$ $75 \%$; and 4, 76-100\%). Patients with immunohistochemical scores $\leq 4$ was considered as non-expression or low-expression, scores $>4$ were defined as the highexpression group. ${ }^{19}$

\section{Statistical Analysis}

The difference of FEN1 expression between osteosarcoma and non-neoplastic bone tissues, as well as the correlation between FEN1 expression and clinicopathological 
parameters, including sex, age, tumor size, tumor location, Enneking stage and chemotherapy response, were analyzed by the $\chi^{2}$ test or Fisher's exact test. Survival analysis was performed by the Kaplan-Meier method, and the differences in survival distributions were compared by the log-rank test. The Cox proportional hazards regression model was used to analyze multivariate survival for the parameters which were found to be significant in the univariate analysis. ${ }^{20}$ All statistical analyses were performed using GraphPad Prism 7.0 (Graph-Pad Software Inc., San Diego, CA, USA) and SPSS 19.0 software (IBM Corp., Armonk, NY, USA). $\mathrm{P}<0.05$ was considered to be significant.

\section{Results}

\section{Clinicopathologic Characteristics of Osteosarcoma Patients}

The clinicopathologic characteristics of osteosarcoma patients are shown in Table 1. A total of 25 males and 15 females with a mean age of 40 years old (range, 5-73 years) were included in this study. Of these patients, twenty-seven patients presented with a tumor size less than $8 \mathrm{~cm}$ and thirteen patients with tumor size more than $8 \mathrm{~cm}$. The location of the tumor included the femur and tibia in 31 patients, and others in 9 patients. According to the Enneking staging system, there were 10 patients classified as stage IIA, and 30 patients classified as stage IIB. Good chemotherapy response could be observed in 14 patients and poor response to chemotherapy could be observed in 26 patients. The mean patient follow-up period was 41.63 months (range, 5-70 months). By the end of the follow-up time, $40 \%(16 / 40)$ of patients survived with no evidence of disease, $22.5 \%(9 / 40)$ of patients remained alive with disease and $37.5 \%(15 / 40)$ of patients succumbed to osteosarcoma.

\section{Immunohistochemical Expression of FENI in Osteosarcoma}

As shown in Figure 1A, the FEN1 was mainly expressed in the cytoplasm of osteosarcoma cells. Of the 40 osteosarcoma patients, 19 (47.5\%) patients presented with FEN1 high expression, while in the non-neoplastic bone specimens, the FEN1 high expression was observed in $10 \%(3 / 30)$, the positive expression rate in osteosarcoma patients was significantly higher than that of nonneoplastic bone specimens (Figure 1B and Table 2, $P<$
0.01). Therefore, FEN1 was overexpressed in osteosarcoma specimens.

\section{Correlation Between FENI Expression in Osteosarcoma and Clinicopathological Characteristics}

The correlations between the expression of FEN1 with clinicopathological parameters, including sex, age, tumor size, tumor location, Enneking stage and chemotherapy response are summarized in Table 3. No significant correlations between the high expression of FEN1 with sex, age, tumor size, tumor location, Enneking stage and

Table I Clinicopathological Characteristics of Osteosarcoma

\begin{tabular}{|c|c|}
\hline Clinicopathologic Data & n (\%) \\
\hline \multicolumn{2}{|l|}{ Sex } \\
\hline Male & $25(62.5)$ \\
\hline Female & $15(37.5)$ \\
\hline Age (years), mean (range) & $40(5-73)$ \\
\hline$>18$ & $26(65.0)$ \\
\hline$\leq 18$ & $14(35.0)$ \\
\hline \multicolumn{2}{|l|}{ Tumor size $(\mathrm{cm})$} \\
\hline$>8$ & $13(32.5)$ \\
\hline$\leq 8$ & $27(67.5)$ \\
\hline \multicolumn{2}{|l|}{ Tumor location } \\
\hline Femur or tibia & $31(77.5)$ \\
\hline Other location & $9(22.5)$ \\
\hline \multicolumn{2}{|l|}{ Enneking staging } \\
\hline$\| \mathrm{A}$ & $10(25.0)$ \\
\hline IIB & $30(75.0)$ \\
\hline \multicolumn{2}{|l|}{ Response to chemotherapy* } \\
\hline Good & $14(35.0)$ \\
\hline Poor & $26(65.0)$ \\
\hline Follow-up duration (month), mean (range) & $41.63(5-70)$ \\
\hline \multicolumn{2}{|l|}{ Survival outcome } \\
\hline Disease-free & $16(40.0)$ \\
\hline Alive with disease & $9(22.5)$ \\
\hline Succumbed to disease & $15(37.5)$ \\
\hline
\end{tabular}

Notes: *Good: tumor necrosis $\geq 90 \%$; poor: tumor necrosis $<90 \%$. 
A

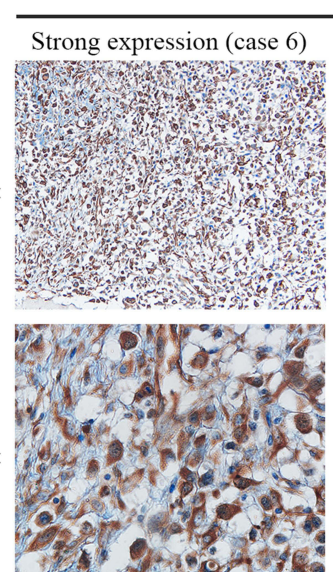

Osteosarcoma

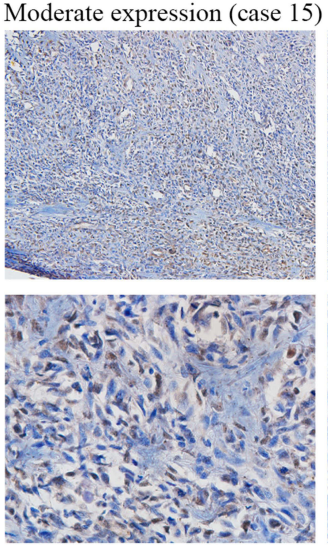

Non-neoplastic bone

Negative expression (case 4)

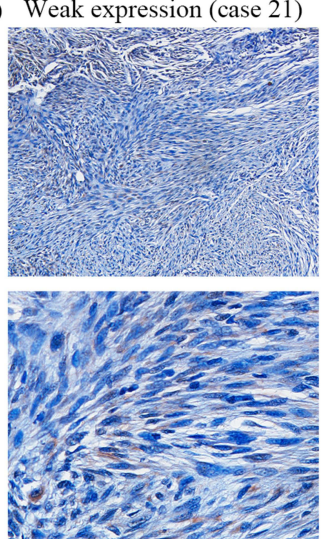

B

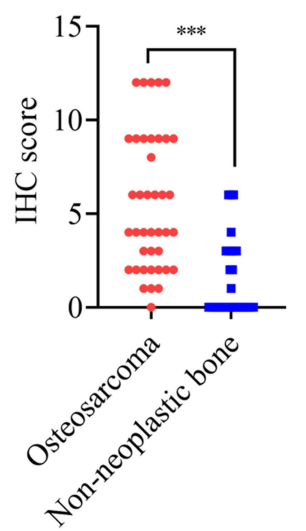

Figure I Immunohistochemical expression of FENI in osteosarcoma and non-neoplastic bone specimens. (A) The different intensities of positive expression in specimens. Scale bars, $100 \mu \mathrm{m}$ (upper) and $25 \mu \mathrm{m}$ (down). (B) The difference of immunohistochemical scores between osteosarcoma group and non-neoplastic bone group. $* * * P<0.00 \mathrm{I}$.

Table 2 FENI Expression in Osteosarcoma and Non-Neoplastic Bone Tissues

\begin{tabular}{|l|c|c|c|}
\hline \multicolumn{5}{|l|}{} & Osteosarcoma, n (\%) & Non-Neoplastic Bone Tissue, n (\%) & P value \\
\hline FENI & $19(47.5)$ & $3(10.0)$ & 0.001 \\
\hline High expression & $21(52.5)$ & $27(90.0)$ & \\
Low expression &
\end{tabular}

Table 3 Association Between FENI Expression and Clinicopathological Characteristics of Osteosarcoma

\begin{tabular}{|c|c|c|c|c|}
\hline & Case Number & High $(n=19)$ & Low $(n=21)$ & $P$ value \\
\hline \multicolumn{5}{|l|}{ Sex } \\
\hline Male & 25 & 11 & 14 & 0.567 \\
\hline Female & 15 & 8 & 7 & \\
\hline \multicolumn{5}{|l|}{ Age (years) } \\
\hline$>18$ & 26 & 13 & 13 & 0.666 \\
\hline$\leq 18$ & 14 & 6 & 8 & \\
\hline \multicolumn{5}{|l|}{ Tumor size $(\mathrm{cm})$} \\
\hline$>8$ & 13 & 6 & 7 & 0.906 \\
\hline$\leq 8$ & 27 & 13 & 14 & \\
\hline \multicolumn{5}{|l|}{ Tumor location } \\
\hline Tibia or femur & 31 & 12 & 19 & 0.092 \\
\hline Other location & 9 & 7 & 2 & \\
\hline \multicolumn{5}{|l|}{ Enneking staging } \\
\hline IIA & 10 & 2 & 8 & 0.100 \\
\hline IIB & 30 & 17 & 13 & \\
\hline \multicolumn{5}{|c|}{ Response to chemotherapy* } \\
\hline Good & 14 & 5 & 9 & 0.273 \\
\hline Poor & 26 & 14 & 12 & \\
\hline
\end{tabular}

Notes: *Good: tumor necrosis $\geq 90 \%$; poor: tumor necrosis $<90 \%$. 
chemotherapy response were observed in this study $(P>0.05)$.

\section{Correlation Between FENI Expression in Osteosarcoma Patient with Clinical Outcome}

To evaluate the independent prognostic ability of FEN1, univariate and multivariate analyses were performed by analyzing the relationship of FEN1 expression with PFS and OS in osteosarcoma patients. Univariate analysis of patient survival as a function of FEN1 expression level is shown at Table 4 and Figure 2. Patients with high FEN1 expression showed shorter mean PFS (12.16 months vs 51.33 months; $P<0.001$, Figure 2E) and OS time (39.04 months vs 61.33 months; $P=0.002$, Figure $2 \mathrm{~F}$ ) when compared with those with low FEN1 expression. In addition to FEN1 expression, Enneking stage (PFS, $P=0.026$, Figure 2A; OS, $P=0.044$, Figure $2 \mathrm{~B}$ ) and chemotherapy response (PFS, $P=0.019$, Figure 2C; OS, $P=0.031$, Figure 2D) were also associated with shorter PFS and OS time upon univariate analysis (Table 4).

Upon multivariate analysis, three clinicopathological factors (FEN1, Enneking stage and chemotherapy response) that were associated with survival time were evaluated. The results showed that high expression of FEN1 independently predicted poor PFS $(\mathrm{HR}=4.73$, $P=0.002)$ and $\mathrm{OS}(\mathrm{HR}=4.01, P=0.038)$ of patients with osteosarcoma (Table 5). Collectively, these findings demonstrated that FEN1 expression was an independent prognostic factor for osteosarcoma patients.

\section{Discussion}

Previous studies have reported that FEN1, as a pivotal factor in DNA replication, long-patch excision repair, and telomere maintenance, has a dual function in DNA replication and repair, and its expression levels and functional disorder can induce genomic instability, leading to cancer development. ${ }^{21-23}$ Some reports have also been reported that overexpression of FEN1 was positively correlated with poor prognosis in several cancers such as breast cancer, ${ }^{24}$ non-small-cell lung, ${ }^{25}$ prostate cancer ${ }^{26}$ and lung adenocarcinoma, ${ }^{27}$ and the high expression of FEN1 was considered to be a prognostic biomarker for these cancers. It is worth mentioning that Liu et al have concluded that FEN1, identified as a key gene associated with OS development, might be a potential biomarker for diagnosis and treatment of this tumor by systematically tracking the altered modules in the reweighted normal and OS PPI networks. ${ }^{28}$ In the present study, we found that osteosarcoma patients, who showed high expression of FEN1, had shorter OS time and PFS time. Meanwhile, multivariate analysis indicated that FEN1 expression was an independent prognostic factor for osteosarcoma patients. Thus, the FEN1 inhibitors, may be considered as the potential target drugs for osteosarcoma treatment.

The relative molecular mechanisms about the role of FEN1 in the progression of several cancers have also been reported by some studies. For example, Zhang et al demonstrated that in hepatocellular carcinoma, FEN1 expression was positively correlated with cell cycle and DNA replication pathway-related genes such as c-Myc, survivin, and cyclin D1, silencing FEN1 could inhibit cell proliferation and migration by regulating expression of these genes. ${ }^{29}$ Becker et al reported that flap endonuclease overexpression was a driver of genome instability in yeast and human cells that impairs DNA replication in a manner dependent on its interaction with PCNA. ${ }^{22}$ Wang et al demonstrated that the FEN1 protein level was tightly controlled by sequential phosphorylation, SUMOylation, and ubiquitination in a cell cycle-dependent manner in breast cancer. Failure of these regulation processes might result in high FEN1 protein level, which was associated with abnormal cell cycle progression and genome instability. ${ }^{30}$ Dong et al indicated that inhibition of IGF-1/miR-610/FEN1 axis could induce OS cells apoptosis. ${ }^{31}$ Therefore, we predicted that the mechanism of FEN1 to affect osteosarcoma progression was achieved by regulating cell cycle and DNA replication of osteosarcoma cells.

Due to the relatively small sample size and short follow-up time in this study, the association of FEN1 protein overexpression with poor prognosis may be bias. Moreover, preoperative neoadjuvant chemotherapy may have influence on the immunohistochemical fidelity of FEN1. Further studies should be focus on the relative molecular mechanism in vivo and in vitro of osteosarcoma.

\section{Conclusion}

In summary, the present study demonstrated that FEN1 was overexpressed in osteosarcoma patients and positively associated with the poor prognosis of osteosarcoma patients. FEN1 could be a promising target for 


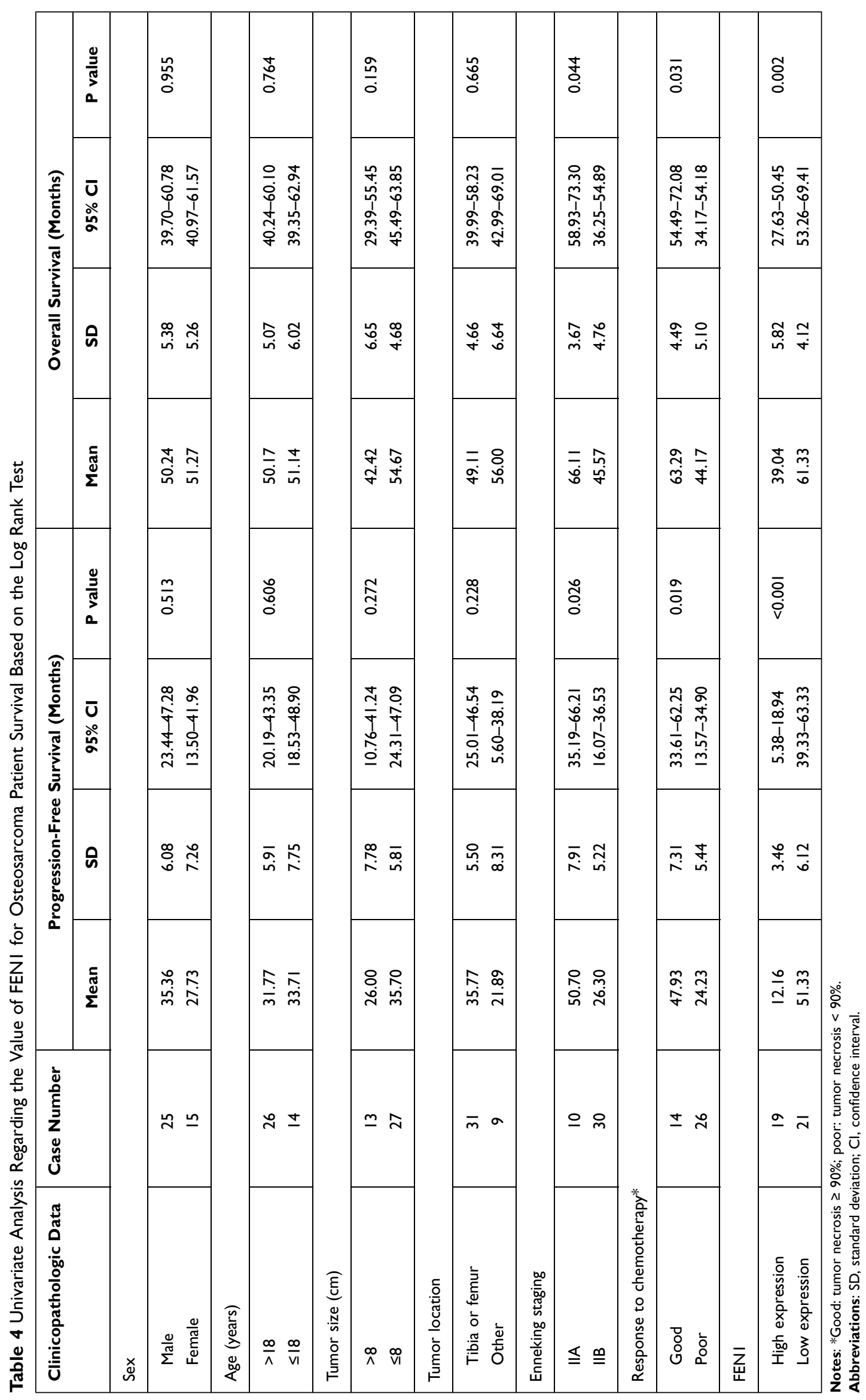



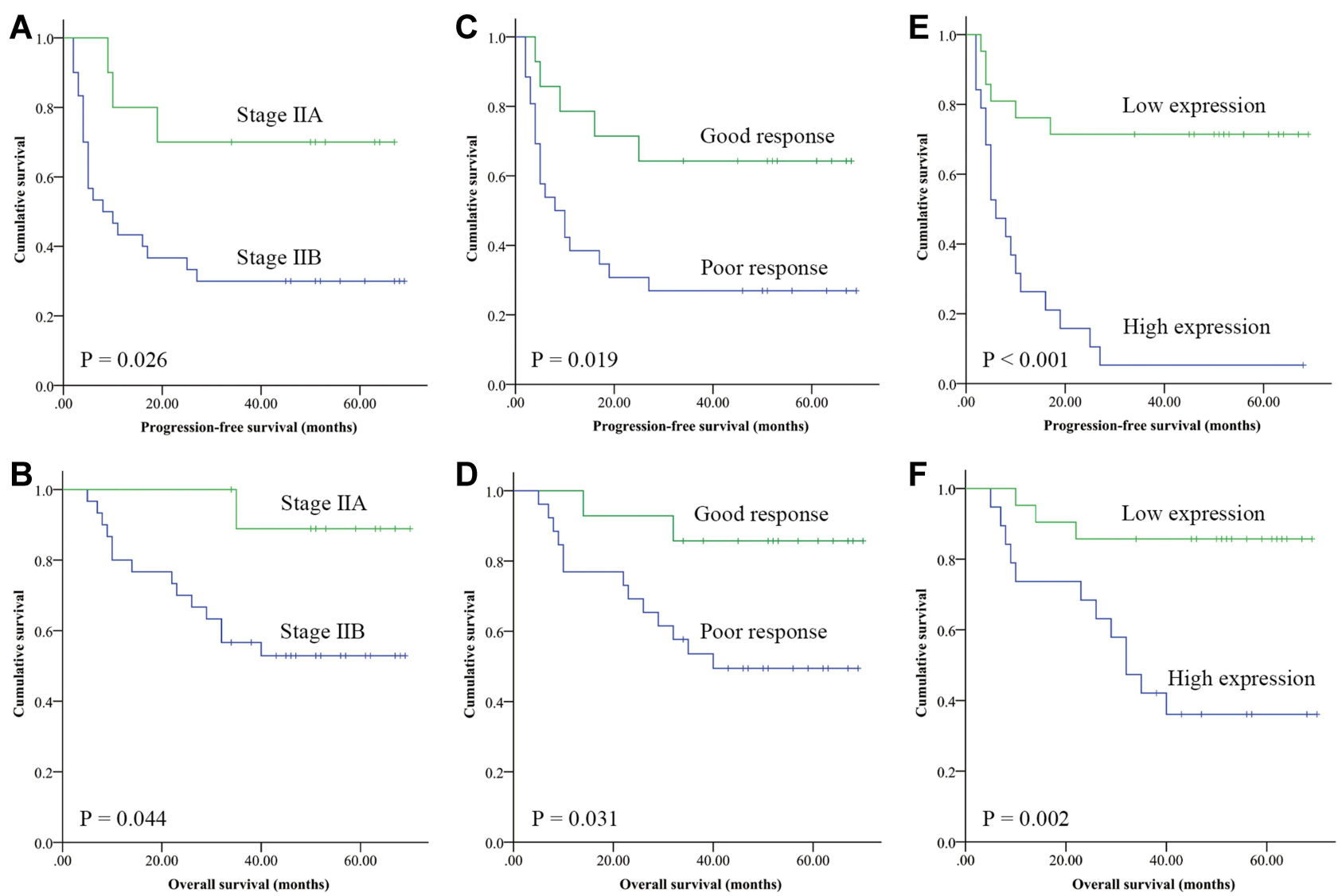

Figure 2 Kaplan-Meier analysis of PFS and OS time by expression of FENI in osteosarcoma patients. Significant differences in PFS $(\mathbf{A}, P=0.026)$ and OS (B, $P=0.044)$ time were observed between patients with Stage IIA and Stage IIB. Significant differences in PFS $(\mathbf{C}, P=0.019)$ and OS $(\mathbf{D}, P=0.03 \mathrm{I})$ time were observed between patients with good chemotherapy response and poor chemotherapy response. Significant differences in PFS $(E, P<0.001)$ and OS $(F, P=0.002)$ time were observed between patients with high FENI expression and low FENI expression.

Abbreviations: PFS, progression-free survival; OS, overall survival.

the survival of osteosarcoma patients if verified in the future. Further study should be focus on the molecular mechanism of FEN1 in the progression of osteosarcoma and exploring the therapeutic value of FEN1 for osteosarcoma.

\section{Ethics Approval and Consent to Participate}

Human Research Committee of the first Affiliated Hospital of Fujian Medical University approved the study. All procedures were performed in accordance with ethical standards laid out in the Declaration of Helsinki. All of the patients were informed of the aims of the study, and written informed consent was obtained.

\section{Consent for Publication}

All authors have read and approved the content, and they agree to submit it for consideration for publication in the journal.

Table 5 Multivariate Analysis Regarding the Value of FENI for Osteosarcoma Patient Survival

\begin{tabular}{|l|l|c|c|c|c|c|c|}
\hline \multirow{2}{*}{ Characteristics } & \multirow{2}{*}{ Comparison } & \multicolumn{2}{|c|}{ Progression-Free Survival (Months) } & \multicolumn{3}{|c|}{ Overall Survival (Months) } \\
\cline { 3 - 8 } & & HR & $\mathbf{9 5 \%} \mathbf{~ I ~}$ & P value & HR & $\mathbf{9 5 \% ~ C I ~}$ & P value \\
\cline { 3 - 8 } & High vs low & 4.73 & $1.76 \sim 12.69$ & 0.002 & 4.01 & $1.08 \sim 14.83$ & 0.038 \\
FENI & IIB vs IIA & 2.38 & $0.67 \sim 8.47$ & 0.178 & 3.67 & $0.45 \sim 29.72$ & 0.223 \\
Response to chemotherapy & Good vs poor & 0.34 & $0.12 \sim 0.92$ & 0.034 & 0.26 & $0.06 \sim 1.15$ & 0.075 \\
\hline
\end{tabular}

Abbreviations: $\mathrm{HR}$, hazard ratio; $\mathrm{Cl}$, confidence interval. 


\section{Acknowledgments}

This work was financially supported by the National Natural Science Foundation of China (81972021), the Joint Funds for the Innovation of Science and Technology in Fujian Province (2019Y9016,2016Y9019), the Outstanding Young Talents Project of the First Affiliated Hospital of Fujian Medical University (YYXQN-ZGX2021), Startup Fund for scientific research, Fujian Medical University (Grant number: 2017XQ1060) (China), the Special Fund of Youth Top-Notch Innovative Talents of Fujian Province (SQNBJ201601).

\section{Disclosure}

Guangxian Zhong and Yunqing Wang are co-first authors for this study. The authors declare that they have no conflicts of interest.

\section{References}

1. Lindsey BA, Markel JE, Kleinerman ES. Osteosarcoma overview. Rheumatol Ther. 2017:4:25-43.

2. Czarnecka AM, Synoradzki K, Firlej W, et al. Molecular biology of osteosarcoma. Cancers. 2020;12:2130.

3. Eaton BR, Schwarz R, Vatner R, et al. Osteosarcoma. Pediatr Blood Cancer. 2021;68(Suppl.2):e28352.

4. Zhang Y, Yang JQ, Zhao N, et al. Progress in the chemotherapeutic treatment of osteosarcoma (Review). Oncol Lett. 2018;16:6228-6237.

5. Kansara M, Teng MW, Smyth MJ, Thomas DM. Translational biology of osteosarcoma. Nat Rev Cancer. 2014;14:722-735.

6. Brown HK, Tellez-Gabriel M, Heymann D. Cancer stem cells in osteosarcoma. Cancer Lett. 2017;386:189-195.

7. Shaikh AB, Li FF, Li M, et al. Present advances and future perspectives of molecular targeted therapy for osteosarcoma. Int $J$ Mol Sci. 2016;17(4):506

8. Liu Y, Kao HI, Bambara RA. Flap endonuclease 1: a central component of DNA metabolism. Annu Rev Biochem. 2004;73:589-615.

9. Balakrishnan L, Bambara RA. Flap endonuclease 1. Аnnu Rev Biochem. 2013;82:119-138.

10. Rossi ML, Bambara RA. Reconstituted Okazaki fragment processing indicates two pathways of primer removal. $J$ Biol Chem. 2006;281:26051-26061

11. He L, Luo L, Zhu H, et al. FEN1 promotes tumor progression and confers cisplatin resistance in non-small-cell lung cancer. Mol Oncol. 2017;11(9):1302-1303.

12. Ma L, Cao X, Wang H, Lu K. Discovery of myricetin as a potent inhibitor of human flap endonuclease 1 , which potentially can be used as sensitizing agent against ht-29 human colon cancer cells. J Agric Food Chem. 2019;67(6):1656-1665.

13. Chen YD, Zhang X, Qiu XG, et al. Functional FEN1 genetic variants and haplotypes are associated with glioma risk. J Neuro-Oncol. 2013;111(2):145-151
14. Zhang K, Keymeulen S, Nelson R, et al. Overexpression of flap endonuclease 1 correlates with enhanced proliferation and poor prognosis of non-small-cell lung cancer. Am J Pathol. 2018;188 (1):242-251.

15. Zeng X, Qu XJ, Zhao CY, et al. FEN1 mediates miR-200a methylation and promotes breast cancer cell growth via MET and EGFR signaling. FASEB J. 2019;33(10):10717-10730.

16. Lam JS, Seligson DB, Yu H, et al. Flap endonuclease 1 is overexpressed in prostate cancer and is associated with a high Gleason score. BJU Int. 2006;98(2):445-451

17. Zhao MS, Ji HJ, Fu Q, Cheng Q, Zhang Y, Yang Y. MicroRNA-134$3 \mathrm{p}$ inhibits ovarian cancer progression by targeting flap structure-specific endonuclease 1 in vitro. Oncol Rep. 2021;45 (1):119-128.

18. Dong SW, Xiao YB, Ma X, et al. miR-193b increases the chemosensitivity of osteosarcoma cells by promoting FEN1-mediated autophagy. Onco Targets Ther. 2019;12:10089-10098.

19. Xu L, Shen JM, Qu JL, et al. FEN1 is a prognostic biomarker for ER + breast cancer and associated with tamoxifen resistance through the ER $\alpha /$ cyclin D1/Rb axis. Ann Transl Med. 2021;9(3):258.

20. Liu Q, Hao L, Lou Z, et al. Survival time and prognostic factors of patients with initial noncurative colorectal liver metastases. Medicine (Baltimore). 2017;96:e8831

21. Li WQ, Hu N, Hyland PL, et al. Genetic variants in DNA repair pathway genes and risk of esophageal squamous cell carcinoma and gastric adenocarcinoma in a Chinese population. Carcinogenesis. 2013;34(7):1536-1542

22. Becker JR, Gallo D, Leung W, et al. Flap endonuclease overexpression drives genome instability and DNA damage hypersensitivity in a PCNA-dependent manner. Nucleic Acids Res. 2018;46 (11):5634-5650

23. Zhao J, Wang G, Del Mundo IM, et al. Distinct mechanisms of nuclease-directed DNA-structure-induced genetic instability in cancer genomes. Cell Rep. 2018;22(5):1200-1210.

24. He L, Zhang Y, Sun H, et al. Targeting DNA flap endonuclease 1 to impede breast cancer progression. eBioMedicine. 2016;14:32-43.

25. Zhang KQ, Keymeulen S, Nelson R, et al. Overexpression of flap endonuclease 1 correlates with enhanced proliferation and poor prognosis of non-small-cell lung cancer. Am $J$ Pathol. 2018;188 (1):242-251.

26. Urbanucci A, Sahu B, Seppälä J, et al. Overexpression of androgen receptor enhances the binding of the receptor to the chromatin in prostate cancer. Oncogene. 2012;31(17):2153-2163.

27. Hwang JC, Sung WW, Tu HP, et al. The overexpression of FEN1 and RAD54B may act as independent prognostic factors of lung adenocarcinoma. PLoS One. 2015;10(10):e0139435.

28. Liu ZZ, Cui ST, Tang B, Wang ZZ, Luan ZX. Identification of key biomarkers involved in osteosarcoma using altered modules. Genet Mol Res. 2016;15(3):gmr.15038277.

29. Zhang YY, Liu X, Liu LW, et al. Upregulation of FEN1 is associated with the tumor progression and prognosis of Hepatocellular carcinoma. Dis Markers. 2020;2020:2514090.

30. Wang JW, Zhou LN, Li Z, et al. YY1 suppresses FEN1 over-expression and drug resistance in breast cancer. BMC Cancer. 2015;15:50.

31. Dong SW, Xiao YB, Zhu ZQ, et al. Metformin sensitizes osteosarcoma cells to chemotherapy through IGF-1R/miR-610/FEN1 pathway. Res Square. 2021. doi:10.21203/rs.3.rs-147084/v1 


\section{Publish your work in this journal}

The International Journal of General Medicine is an international, peer-reviewed open-access journal that focuses on general and internal medicine, pathogenesis, epidemiology, diagnosis, monitoring and treatment protocols. The journal is characterized by the rapid reporting of reviews, original research and clinical studies across all disease areas. The manuscript management system is completely online and includes a very quick and fair peer-review system, which is all easy to use. Visit http://www.dovepress.com/ testimonials.php to read real quotes from published authors.

Submit your manuscript here: https://www.dovepress.com/international-journal-of-general-medicine-journal 